\title{
Iron, infection and immune function
}

\author{
BY CHAIM HERSHKO \\ Department of Medicine, Shaare Zedek Medical Center and Department of Nutrition, \\ Hebrew University Hadassah Medical School, PO Box 293, Jerusalem, Israel
}

A decrease in circulating $\mathrm{Fe}$, or hypoferraemia, is one of the most constant features of infectious disease. Since Fe deprivation in bacterial cultures is regularly associated with inhibition of growth, it has been suggested that $\mathrm{Fe}$ deficiency may represent an important defence mechanism (Weinberg, 1990). The term 'nutritional immunity' has been introduced by Kochan (1973) to underline the importance of Fe deprivation as a key mechanism limiting the growth of invading organisms. Interleukin-1 (IL-1), a protein released by mononuclear phagocytes in response to microbial invasion, is a key mediator in the inflammatory reaction and is directly responsible for the hypoferraemia of inflammation (Dinarello, 1984). It enhances the synthesis of a number of acute-phase proteins such as fibrinogen, haptoglobin, ceruloplasmin, amyloid A protein and ferritin (Fig. 1). The result of increased ferritin synthesis is a block in Fe release resulting in reduced serum Fe levels.

Because of the paucity of clinical information supporting the significance of $\mathrm{Fe}$ deficiency or overload in determining the severity of infectious disease in man, the nutritional immunity hypothesis has remained a topic of continued controversy (Hershko \& Peto, 1988). This controversy is of more than academic interest, since both $\mathrm{Fe}$ deficiency and infectious diseases are common conditions, and Fe supplementation in some populations may resolve one problem while aggravating the other. In the text that follows, I shall discuss briefly the importance of microbial $\mathrm{Fe}$ requirements, the role of $\mathrm{Fe}$

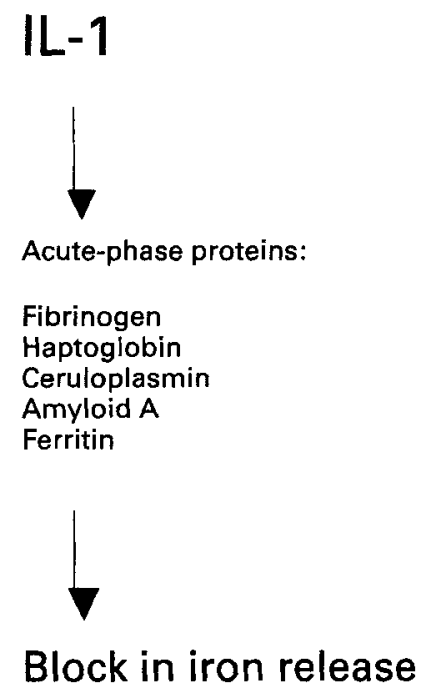

Fig. 1. Effect of interleukin-1 on iron release through increased production of acute-phase reacting proteins. 
in host resistance, the effect of Fe deficiency and overload on clinical infection and the control of infection by selective Fe deprivation through Fe-chelating therapy.

\section{MICROBIAL IRON REQUIREMENTS}

An essential requirement of pathogens for their continued multiplication in the body is the acquisition of sufficient $\mathrm{Fe}$ for their growth. Most micro-organisms obtain $\mathrm{Fe}$ by producing their own Fe-binding compounds, siderophores, classified chemically as either phenolates or hydroxamates (Neilands, 1981). These siderophores are able to compete successfully with the Fe-binding proteins of the host.

The importance of Fe for microbial infection is illustrated by the close correlation between the availability of $\mathrm{Fe}$ and bacterial virulence (Payne, 1988). For example, in both Escherichia coli and Vibrio, plasmids encoding for Fe-sequestration systems are also determinants of virulence (Crosa, 1984). The presence of a siderophore-binding protein on the cell surface of Pseudomonas aeruginosa is essential for its virulence (Sokol, 1987). Neisseriae are able to develop surface receptors specific for human transferrin and lactoferrin in response to Fe starvation, and only Neisseriae utilizing transferrin-Fe are pathogenic (Schryvers \& Gonzalez, 1989). Finally, injection of inorganic Fe enhances the in vivo virulence of Klebsiella, Pseudomonas, E. coli and a large number of other bacteria (Sussman, 1974).

In contrast, in some cases Fe deprivation promotes the virulence of pathogens. Thus, Corynebacterium diphtheria, Clostridium tetani, Shigella and Psuedomonas produce their toxins optimally under conditions of Fe deprivation (Masawe \& Nsanzumhire, 1973). Indeed, the use of Fe salts for the local treatment of diphtherial pharyngitis was a common practice in the 19 th century. Thus, instead of limiting infection, in some cases Fe deficiency may actually lead to the emergence of more virulent bacteria and potentially more serious disease.

\section{IRON AND CELLULAR RESISTANCE}

The relationship between $\mathrm{Fe}$ status and immune function and cellular resistance to infection has been the subject of a number of extensive recent reviews (Dallman, 1987; Good et al. 1988; Dhur et al. 1989). Studies in leucocytes obtained from Fe-deficient subjects have shown a number of abnormalities (Table 1). In Fe-deficient neutrophils bactericidal activity was reduced (Walter et al. 1986) and the respiratory burst was impaired (Murakawa et al. 1987). In Fe-deficient lymphocytes activation and effector function were normal, but the proliferative response to mitogens was reduced (Brock \& Mainou-Fowler, 1983). Antibody-mediated immunity in Fe-deficient individuals appeared to be normal (Dallman, 1987).

Table 1. Iron deficiency and host resistance

\begin{tabular}{lll}
\hline \hline Cell & Effect of deficiency & Reference \\
\hline Neutrophils & Decreased bactericidal activity & Walter et al. (1986) \\
& Impaired respiratory burst activity & Murakawa et al. (1987) \\
Lymphocytes & Impaired response to mitogens & Brock \& Mainou-Fowler (1983) \\
\hline
\end{tabular}


Abnormal function of both neutrophils and lymphoctyes has been encountered in patients with Fe overload. Neutrophils from patients with transfusional siderosis showed severe impairment of phagocytosis, and myeloperoxidase activity inversely correlated with serum ferritin concentrations. This could be reversed by several months of treatment with desferrioxamine (DF) in the Fe-loaded hosts (Waterlot et al. 1985; Cantinieaux et al. 1987). In another study, impaired activity of natural killer cells against target K562 cells has been shown in lymphocytes obtained from thalassaemic patients. This abnormality was in direct proportion to the severity of transfusional Fe overload in the donor, and was corrected by in vitro incubation with DF, implying that the functional impairment was a direct consequence of Fe overload (Hilgartner \& Grady, 1986). Reduced responsiveness of splenic mononuclear cells to various mitogens has been found in thalassaemic mice (Ampel et al. 1989). These observations indicate that both $\mathrm{Fe}$ deficiency and $\mathrm{Fe}$ overload may result in impaired function of cells associated with host defence in microbial infection, and that a normal Fe status is probably the optimal condition allowing a full phagocytic and immune response to pathogens.

\section{CLINICAL STUDIES IN IRON DEFICIENCY AND OVERLOAD}

Clinical studies in which the incidence and severity of infectious diseases are correlated with $\mathrm{Fe}$ status, are of great value in assessing the validity of the nutritional immunity hypothesis. In the following, an attempt will be made to evaluate the available information relevant to this question.

\section{(1) Chronic iron deficiency}

As emphasized in a recent review by Dallman (1987), there are a number of inherent limitations to the study of infection in Fe deficiency: socio-economic status, additional nutritional deficiencies, poor housing conditions, exclusion of severe anaemia, misinterpretation of low serum Fe. A close relationship exists between poverty and the prevalence of $\mathrm{Fe}$ deficiency, and direct comparison between the incidence of infectious diseases in an Fe-deficient and that of an Fe-replete population may be meaningless because of many other differences between these populations such as socio-economic status, the coexistence of additional nutritional deficiencies unrelated to Fe deficiency, and housing conditions. In addition, subjects with severe Fe-deficiency anaemia must be excluded from prospective randomized studies because of ethical considerations, thus, restricting the study to moderate to mild deficiency and possibly obscuring the effect of Fe deficiency. Finally, both anaemia and reduced transferrin saturation may be caused by infection and, unless strict criteria are applied, may result in an overdiagnosis of $\mathrm{Fe}$ deficiency associated with infection.

Because of these considerations, it is preferable to study the effect of Fe administration on the incidence and severity of infection, comparing the results with untreated controls with an identical background. Unfortunately, there are only a few studies of this sort, and most of them represent uncontrolled observations. A distinction should be made between oral and parenteral colloidal Fe therapy, since the massive deposition of colloidal (parenteral) $\mathrm{Fe}$ in the reticulo-endothelial (RE) system may result in its temporary blockade.

Parenteral iron therapy: Most of these reports originate from tropical countries. Barry 
Table 2. Effect of iron therapy on infection

\begin{tabular}{|c|c|c|}
\hline & Effect of therapy & Reference \\
\hline Western countries & $\begin{array}{l}\text { No effect on rates of infection } \\
\text { Decreased incidence of infection }\end{array}$ & $\begin{array}{l}\text { Burman (1972) } \\
\text { Andelman \& Sered (1966) }\end{array}$ \\
\hline Tropical countries & $\begin{array}{l}\text { Increased incidence of malaria, brucella, } \\
\text { tuberculosis } \\
\text { No effect on respiratory and enteric infections } \\
\text { No effect on prevalence or severity of malaria }\end{array}$ & $\begin{array}{l}\text { Murray et al. (1978) } \\
\text { Damsdaran et al. (1979) } \\
\text { Harvey et al. (1989) }\end{array}$ \\
\hline
\end{tabular}

\& Reeve (1977) have reported an increased incidence of neonatal sepsis among Polynesian infants following the introduction of a prophylactic programme of intramuscular iron dextran therapy, and a decrease from 22 to 2 per 1000 in the incidence of sepsis after discontinuation of the programme. Unfortunately, the incidence of neonatal sepsis in this population before parenteral Fe therapy is unknown, although mortality rates increased after initiation of treatment (Barry \& Reeve, 1988). As the entire population had been treated, there was no control population left for direct comparison.

The effect of total dose intravenous iron dextran infusion was studied among Tanzanian women in another uncontrolled study by Byles \& D'sa (1970). The incidence of post-infusion malaria was $2.8 \%$, but in the absence of untreated controls the proportion of subjects who might have developed parasitaemia without Fe treatment is unknown. More recently, a prospective, randomized longitudinal study has been performed among infants in Papua New Guinea to study the effect of intramuscular iron dextran given prophylactically at the age of 2 months (Oppenheimer et al. 1986). Within the following year the prevalence of slide-positive malaria was about $50 \%$ higher in the Fe-treated group, but there was no increase in the density of parasitaemia or rates of clinical malaria. In contrast to the study reported by Barry \& Reeve (1988), there was no difference in the rate of fatal infections between the two groups. Moreover, in a study by Cantwell (1972) in which Maori newborn infants were randomly selected to receive iron dextran injections, a small but statistically significant reduction in hospitalization rates has been observed in the Fe-treated group.

The unphysiological manner of Fe delivery is a serious limitation of studies involving the parenteral use of colloidal Fe. Parenteral Fe therapy may result in serum Fe concentrations exceeding temporarily the total Fe-binding capacity of transferrin, and the massive deposition of colloidal Fe in the RE system may interfere with its protective function in a manner totally unrelated to Fe nutrition. Thus, although such studies may provide some information on the risks involved in the use of colloidal $\mathrm{Fe}$, they bear little relevance to the question of nutritional immunity.

Oral iron therapy: Unlike parenteral $\mathrm{Fe}$ therapy, treatment with oral $\mathrm{Fe}$ medications is more physiological and does not involve the risks of extremely high serum $\mathrm{Fe}$ concentrations or interference with RE function (Table 2). Several large-scale studies were conducted among children in Western industrialized countries in which the effect of long-term Fe supplementation on infection has been examined in a prospective manner. Two of these studies were randomized. Most problems encountered were common respiratory and gastrointestinal infections (Mackay, 1928; Andelman \& Sered, 1966; Burman, 1972). One study showed no differences between the Fe-treated and control 
groups and the other two showed a decreased incidence of infections following $\mathrm{Fe}$ treatment.

Oral Fe treatment in tropical countries represents a special category of studies since the incidence and severity of infectious diseases in this environment may be much greater than in Western countries, and also because most of the preliminary reports of uncontrolled studies of parenteral Fe treatment aggravating infection originated from such countries. In a prospective randomized study of adult Somali nomads with Fe-deficiency anaemia (Murray et al. 1978), there was a twelvefold increase in episodes of infection in patients receiving Fe compared with untreated controls. The most striking differences were in malaria, brucellosis and tuberculosis. However, these dramatic effects of oral Fe treatment on tropical infections have not been confirmed by other randomized studies in similar populations. Thus, in a study conducted among preschool village children in India, no significant increase in the frequency or duration of respiratory and enteric infections has been found after 12 months of Fe supplementation despite careful follow-up by weekly home visits (Damsdaran et al. 1979). Likewise, in a recent prospective randomized study of prepubescent schoolchildren in Papua New Guinea receiving oral Fe treatment, there was no difference in malarial parasite rate, parasite density, or levels of anti-malarial IgG between children receiving oral $\mathrm{Fe}$ and the control group (Harvey et al. 1989).

It is difficult to reconcile these seemingly conflicting observations on the effect of $\mathrm{Fe}$ therapy on infection. Some of the earlier reports are uncontrolled anecdotal observations. Most of the carefully designed prospective randomized trials show no conclusive evidence of an increased risk of infection following oral Fe treatment. It is recommended that in order to evaluate the effect of Fe, parenteral Fe preparations should be avoided, since this mode of therapy may introduce additional variables unrelated to the effect of $\mathrm{Fe}$ per se. In view of the importance of the inter-relationship between $\mathrm{Fe}$ administration and the risk of infection, additional studies exploring this question in various populations are greatly needed.

\section{(2) Chronic iron overload}

The most important clinical conditions in which Fe overload has been claimed to be responsible for an increased incidence of infections are idiopathic haemochromatosis, chronic renal failure and thalassaemia major. However, it is difficult to distinguish the effect of the underlying morbidity associated with these clinical entities from the direct effect of $\mathrm{Fe}$ on infection.

Infection is not a significant problem in the precirrhotic phase of idiopathic haemochromatosis. Mortality among terminal patients is mainly caused by hepatic or cardiac failure, hepatoma and diabetes (Finch \& Finch, 1955). Pneumonia is a terminal event in $12 \%$ of patients, but with coexistent cirrhosis and diabetes this can hardly be attributed to $\mathrm{Fe}$ alone.

In patients maintained on chronic haemodialysis there is an increased incidence and severity of bacterial infections. Several investigators have shown that infection is more common in patients with increased ferritin levels (Seifert et al. 1987; Tielemans et al. 1989) and it was suggested that overload may be the main cause of the observed increased susceptibility of haemodialysis patients to bacterial infection. However, the observed ferritin values ranging from 500 to $2000 \mu \mathrm{g} / \mathrm{l}$ are much lower than the range of 
values observed in other conditions associated with severe transfusional Fe overload. It is quite likely that the increased transfusion requirement of these patients is indicative of an increased severity of the underlying disease and that there is no cause-and-effect relationship between increased ferritin measurements and infection. With the introduction of regular erythropoietin support to such patients it would be interesting to examine whether such treatment, leading to improved haemoglobin and reduced ferritin levels, may also result in a reduced susceptibility to infection.

Severe infections are responsible for about $20 \%$ of deaths in homozygous $\beta$-thalassaemia. Since transfusional Fe overload is a major problem in many of these patients, thalassaemia appears to be an important illustration of the role of $\mathrm{Fe}$ overload in infection. However, a close examination of risk factors of infection in thalassaemia shows that other variables may be more important. Among patients in two major series of studies reported from the USA and Britain, all but two fatal infections and all severe infections such as meningitis, peritonitis and osteomyelitis occurred in splenectomized patients (Smith et al. 1964; Modell \& Berdoukas, 1984). The increased risk of infection in splenectomized thalassaemic patients may be explained by a reduced response to circulating antigens and the reduced clearance of bacteria associated with asplenia (Bullen \& Losowsky, 1979). Anaemia is a second important risk factor of infection in thalassaemia. In the British series of studies, the incidence of pneumonia in anaemic patients was eight times higher than that in well-transfused patients, with haemoglobins ranging from 100 to $140 \mathrm{~g} / \mathrm{l}$. In contrast, the risk of infection in thalassaemic patients is unrelated to age, which is an indirect indicator of transfused Fe burden, or to the severity of Fe overload. Of the ten thalassaemic patients with fatal infections described by Modell \& Berdoukas (1984), four were well-chelated patients and in three others the total estimated Fe load was less than $5 \mathrm{~g}$. Thus, although infection is an important complication of thalassaemia, there is no convincing evidence that Fe overload has a major role in its causation.

\section{EFFECT OF IRON CHELATION}

Since pathogens are dependent on Fe for their unimpeded growth, it has been suggested that Fe-chelating drugs may be effective in controlling infection (Table 3). Several recent studies have shown that this may be feasible. In vitro and in vivo studies with Trypanosoma cruzi (Lalonde \& Holbein, 1986), Pneumocystis carinii (Clarkson et al. 1990), Legionella pneumophila (Byrd \& Horwitz, 1989), Plasmodium falciparum, Plasmodium vinckei, and Plasmodium berghei in mice, rats and monkeys (Fritsch et al. 1985; Hershko \& Peto, 1988; Pollack et al. 1987) have shown that it is possible to inhibit the proliferation of these micro-organisms by the Fe-chelating agent DF. Subsequent in vivo studies with $P$. berghei have shown that parasite inhibition is independent of host Fe status, and that the therapeutic effect of DF is explained by its direct interaction with a chelatable labile $\mathrm{Fe}$ pool within the infected erythrocyte. It is assumed that parasite inhibition is caused by inactivation of ribonucleotide reductase, an Fe-dependent rate-limiting enzyme in DNA synthesis (Hoffbrand et al. 1976). Since the ability of DF to penetrate the erythrocyte membrane is limited, other Fe-chelating compounds with a higher lipid solubility have been studied and several of these have shown improved anti-malarial activity (Hershko et al. 1991). Preliminary studies in patients with asymptomatic $P$. falciparum parasitaemia, and in children with cerebral malaria con- 


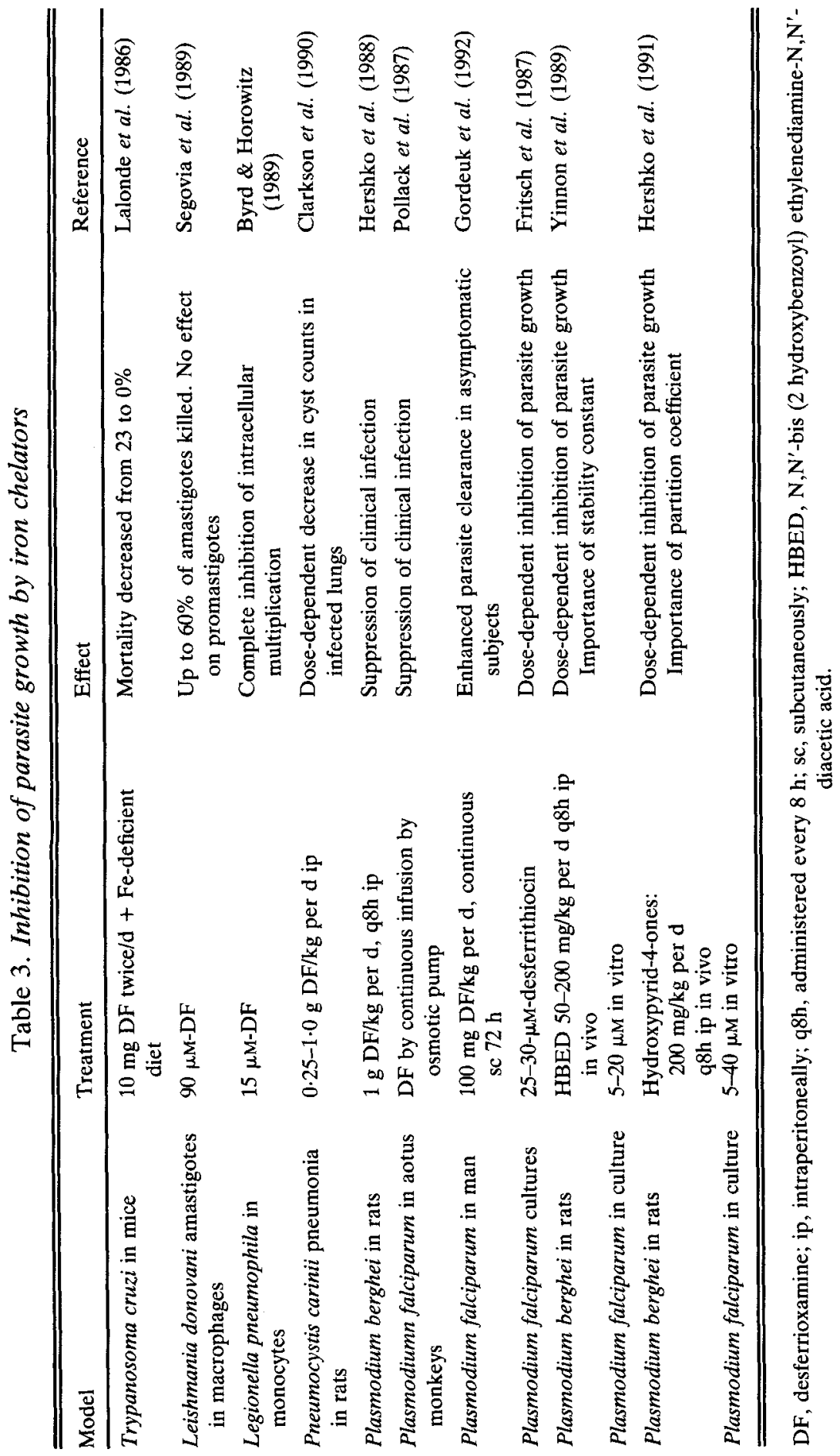


ducted in Zambia (Gordeuk et al. 1992) have shown that DF given by continuous infusion at $100 \mathrm{mg} / \mathrm{kg}$ per $\mathrm{d}$ for $72 \mathrm{~h}$ results in the complete disappearance of parasites from the circulation, and a significant shortening of the duration of altered consciousness.

Studies in human monocytes activated by L. pneumophila and in mouse macrophages treated with $\gamma$-interferon have shown that the availability of $\mathrm{Fe}$ for invading organisms is further limited by down-regulating the number of transferrin receptors on the cellular surface (Byrd \& Horwitz, 1989; Lane et al. 1991). In addition, the sharp increase in apoferritin synthesis induced by inflammation may result in a shift of labile intracellular Fe to the relatively unavailable compartment of ferritin-Fe stores (Konijn \& Hershko, 1977). Thus, the alterations in Fe homeostasis associated with inflammation may limit the availability of intracellular $\mathrm{Fe}$ for invading organisms by reducing transferrin-Fe uptake as well as by diverting the labile $\mathrm{Fe}$ pool into relatively unavailable storage compartments. Fe chelators simulate this intracellular protective mechanism. Comparison of all available in vitro studies on the antimicrobial effect of DF and other chelators reveals a striking similarity in the inhibitory concentration of these drugs. Without exception, the inhibitory concentration is about $20 \mu \mathrm{M}$, and maximal effect is achieved between 40 and $100 \mu \mathrm{M}$. These values may represent the approximate magnitude of the intracellular chelatable Fe pool.

The antimicrobial effect of Fe-chelating drugs lends new meaning to the term nutritional immunity and opens new channels for exploring the possibility of controlling infection by means of selective intracellular Fe deprivation. Experimental models for studying the effect of Fe chelators on other intracellular pathogens such as Toxoplasma gondii, Chlamydia psittachi, Mycobacterium tuberculosis and Leishmania spp. should be established. Packaging the chelator in liposomes or erythrocyte ghosts, or manipulating their lipid solubility to improve their delivery to appropriate target organs such as the macrophage system may greatly improve their efficiency. In view of its short half-life and poor oral effectiveness it is unlikely that DF per se will be suitable for clinical use as a practical antimicrobial agent. However, with the introduction of simple, orally effective new chelators, it is reasonable to expect that future research may lead to the identification of $\mathrm{Fe}$ chelators with considerable usefulness in the control of infectious disease.

\section{REFERENCES}

Ampel, N. M., Van Wyck, D. B., Aquirre, M. L., Willis, D. G. \& Popp, R. A. (1989). Resistance to infection in murine beta-thalassemia. Infection and Immunity 57, 1011-1017.

Andelman, M. B. \& Sered, B. R. (1966). Utilization of dietary iron by term infants: A study of 1048 infants from a low socioeconomic population. American Journal of Disease in Children 111, 45-55.

Barry, D. M. J. \& Reeve, A. W. (1977). Increased incidence of gram-negative neonatal sepsis with intramuscular iron administration. Pediatrics 60, 908-912.

Barry, D. M. J. \& Reeve, A. W. (1988). Iron and infection. British Medical Journal 296, 1736.

Brock, J. H. \& Mainou-Fowler, T. (1983). The role of iron and transferrin in lymphocyte transformation. Immunology Today 4, 347-351.

Bullen, A. W. \& Losowsky, M. S. (1979). Consequences of impaired splenic function. Clinical Science 57, 129-137.

Burman, D. (1972). Hemoglobin levels in normal infants aged 3-24 months and the effect of iron. Archives of Disease in Childhood 47, 261-271.

Byles, A. B. \& D'sa, A. (1970). Reduction of reaction due to iron dextran infusion using chloroquine. British Medical Journal 3, 625-627. 
Byrd, T. F. \& Horwitz, M. A. (1989). Interferon gamma-activated human monocytes downregulate transferrin receptors and inhibit the intracellular multiplication of Legionella pneumophila by limiting the availability of iron. Journal of Clinical Investigation 83, 1457-1465.

Cantinieaux, B., Hariga, C., Ferster, A., De Maertelaere, E., Toppet, M. \& Fondu, P. (1987). Neutrophil dysfunctions in thalassaemia major: the role of cell iron overload. European Journal of Haematology 39, 28-34.

Cantwell, R. J. (1972). Iron deficiency anaemia in infancy. Clinical Pediatrics 11, 443-449.

Clarkson, A. B., Saric, M. \& Grady, R. W. (1990). Deferoxamine and eflornitine (DF- $\alpha$-difluoromethylornithine) in a rat model of Pneumocystis carinii pneumonia. Antimicrobial Agents and Chemotherapy 34, $1833-1835$.

Crosa, J. H. (1984). The relationship of plasmid mediated iron transport and bacterial virulence. Annual Review of Microbiology 38, 69-89.

Dallman, P. R. (1987). Iron deficiency and the immune response. American Joumal of Clinical Nutrition 46, 329-334.

Damsdaran, M., Naidu, A. N. \& Sarma, K. V. R. (1979). Anemia and morbidity in rural preschool children. Indian Journal of Medical Research 69, 448-456.

Dhur, A., Galan, P. \& Hercberg, S. (1989). Iron status, immune capacity and resistance to infections. Comparative Biochemistry and Physiology 94, 11-19.

Dinarello, C. A. (1984). Interleukin-1. Review of Infectious Diseases 6, 51-95.

Finch, S. C. \& Finch, C. A. (1955). Idiopathic hemochromatosis, an iron storage disease. Medicine 34, $381-430$.

Fritsch, G., Sawatzki, G., Treumer, J., Jung, A. \& Spira, D. T. (1987). Plasmodium falciparum: Inhibition in vitro with lactoferrin, desferrithiocin, and desferricrocin. Experimental Parasitology 63, 1-9.

Fritsch, G., Treumer J., Spira. D. T. \& Jung, A. (1985). Plasmodium vinckei: suppression of mouse infections with desferrioxamine B. Experimental Parasitology 60, 171-174.

Good, M. F., Powell, L. W. \& Halliday, J. W. (1988). Iron status and cellular immune competence. Blood Reviews 2, 43-49.

Gordeuk, V. R., Thuma, P. E., Brittenham, G. M., Zulu, S., Simwanza, G., Mhangu, A., Flesch, G. \& Parry, D. (1992). Iron chelation with deferoxamine $B$ in adults with asymptomatic Plasmodium falciparum parasitemia. Blood 79, 308-312.

Harvey, P. W., Heywood, P. F., Nesheim, M. C., Galme, K., Zegans, M., Habicht, J. P., Stephenson, L. S., Radimer, K. L., Brabin, B. \& Forsyth, K. (1989). The effect of iron therapy on malarial infection in Papua New Guinean schoolchildren. American Journal of Tropical Medicine and Hygiene 40, 12-18.

Hershko, C. \& Peto, T. E. A. (1988). Deferoxamine inhibition of malaria is independent of host iron status. Journal of Experimental Medicine 168, 375-387.

Hershko, C., Peto, T. E. A. \& Weatherall, D. J. (1988). Regular review. Iron and infection. British Medical Journal 296, 666-667.

Hershko, C., Theanacho, E. N., Spira, D. T., Peter, H. H., Dobbin, P. \& Hider, R. C. (1991). The effect of $\mathrm{N}$-alkyl modification on the antimalarial activity of 3-hydroxypyrid-4-one oral iron chelators. Blood 77 , 637-643.

Hilgartner, M. W. \& Grady, R. W. (1986). Decreased natural killer activity in thalassemia major: a possible consequence of iron overload. Journal of Immunology 136, 1635-1640.

Hoffbrand, A. V., Ganeshaguru, K., Hooton, J. W. L. \& Tattersall, M. H. N. (1976). Effect of iron deficiency and desferrioxamine on DNA synthesis in human cells. British Journal of Haematology 33, 517-526.

Kochan, I. (1973). The role of iron in bacterial infections with special consideration of host-tubercle bacillus interaction. Current Topics in Microbiology and Immunology 60, 1-30.

Konijn, A. M. \& Hershko, C. (1977). Ferritin synthesis in inflammation: 1. Pathogenesis of impaired iron release. British Journal of Haematology 37, 7-16.

Lalonde, R. G. \& Holbein, B. E. (1986). Role of iron in Trypanosoma cruzi infection in mice. Journal of Clinical Investigation 73, 470-476.

Lane, T. E., Wu-Hsieh, B. A. \& Howard, D. H. (1991). Iron limitation and the gamma interferon-mediated antihistoplasma state of murine macrophages. Infection and Immunity 59, 2274-2278.

Mackay, H. M. (1928). Anemia in infancy: its prevalence and prevention. Archives of Disease in Childhood 3, 117-147.

Masawe, A. E. J. \& Nsanzumhire, H. (1973). Growth of bacteria in vitro in blood from patients with severe iron deficiency anemia and from patients with sickle cell anemia. American Journal of Clinical Pathology 59, 706-711. 
Modell, B. \& Berdoukas, V. (1984). The Clinical Approach to Thalassaemia. London: Grune and Stratton.

Murakawa, H., Bland, C. E., Willis, W. T. \& Dallman, P. R. (1987). Iron deficiency and neutrophil function: different rates of correction of the depressions in oxidative burst and myeloperoxidase activity after iron treatment. Blood 69, 1464-1468.

Murray, M. J., Murray, A. B., Murray, M. B. \& Murray, C. J. (1978). The adverse effect of iron repletion on the course of certain infections. British Medical Journal 2, 1113-1115.

Neilands, J. B. (1981). Microbial iron compounds. Annual Review of Biochemistry 50, 715-731.

Oppenheimer, S. J., Gibson, F. D. \& McFarlane, S. B. (1986). Iron supplementation increases prevalence and effects of malaria: report on clinical studies in Papua New Guinea. Transcripts of Royal Society of Tropical Medicine and Hygiene 80, 603-612.

Payne, S. M. (1988). Iron and virulence in the family of Enterobacteriaceae. Critical Reviews in Microbiology 16, 81-111.

Pollack, S,, Rossan, R. N., Davidson, D. E. \& Escajadillo, A. (1987). Desferrioxamine suppresses Plasmodium falciparum in aotus monkeys. Proceedings of the Society of Experimental Biology and Medicine 184, 162-165.

Schryvers, A. B. \& Gonzalez, G. C. (1989). Comparison of the abilities of different protein sources of iron to enhance Neisseria meningitidis infection in mice. Infection and Immunity 57, 2425-2429.

Segovia, M., Navarro, A. \& Artero, J. M. (1989). The effect of liposome-entrapped desferrioxamine on Leishmania donovani in vitro. Annals of Tropical Medicine and Parasitology 83, 357-360.

Seifert, A., von Herrath, D. \& Schaefer, K. (1987). Iron overload, but not treatment with desferrioxamine favours the development of septicemia in patients on maintenance hemodialysis. Quarterly Journal of Medicine 65, 1015-1024.

Smith, C. H., Erlandson, M. E., Stern, G. \& Hilgartner, M. W. (1964). Post splenectomy infection in Cooley's anemia. Annals of New York Academy of Sciences 119, 748-758.

Sokol, P. A. (1987). Surface expression of ferripyochelin-binding protein is required for virulence of Pseudomonas aeruginosa. Infection and Immunity 55, 2021-2025.

Sussman, M. (1974). Iron and infection. In Iron in Biochemistry and Medicine, pp. 649-679 [A. Jacobs \& M. Worwood, editors]. New York: Academic Press.

Tielemans, C. L., Lenclud, C. M., Wens, R., Collart, F. E. \& Dratwa, M. (1989). Critical role of iron overload in the increased susceptibility of haemodialysis patients to bacterial infections. Beneficial effects of desferrioxamine. Nephrology Dialysis and Transplantation 4, 883-887.

Walter, T., Arredondo, S., Arevalo, M. \& Stekel, A. (1986). Effect of iron therapy on phagocytosis and bactericidal activity in neutrophils of iron-deficient infants. American Journal of Clinical Nutrition 44, 877-882.

Waterlot, Y., Cantinieaux, B., Hariga-Muller, C., DeMaertelaere-Laurent, C., Vanherweghem, J. L. \& Fondu, P. (1985). Impaired phagocytic activity of neutrophils in patients receiving haemodialysis: the critical role of iron overload. British Medical Journal 291, 501-504.

Weinberg, E. D. (1990). Cellular iron metabolism in health and disease. Drug Metabolism Reviews 22 , 531-579.

Yinnon, A. M., Theanacho, E. N., Grady, R. W., Spira, D. Y. \& Hershko, C. (1989). Antimalarial effect of HBED and other phenolic and catecholic iron chelators. Blood 74, 2166-2171. 